\title{
Overcoming Jet Lag: Optimizing Aerosol Delivery With and Without Jet Nebulizers
}

Early studies have shown that only a small fraction of the nominal nebulizer dose $(1-12 \%)$ is available for delivery to the lungs in intubated children and adults..$^{1-3}$ Since then, the characterization of forces governing aerosol delivery in ventilated patients has led to improvements in techniques, and the development of newer aerosol generators with the ability to deliver as much drug to ventilated patients as seen in the ambulatory setting. ${ }^{4}$ Optimizing aerosol nebulization in mechanically ventilated patients requires careful consideration of several factors that affect aerosol drug delivery. Major factors identified in the literature include nebulizer type and position within the circuit, timing of nebulization with respect to respiratory cycle, and humidification of the inspired gas. ${ }^{5}$ However, despite advances in the field, there is still no agreed upon or universally recommended method of aerosol delivery to ventilated patients. This may be due in part to difficulty reconciling disparities among various studies. The lack of standardization, and the introduction of numerous variables known to influence aerosol delivery, such as endotracheal tube size, tidal volumes, loading volume, inspiratory flow rates, bias flow, the external flow to jet nebulizers, and the overall volume of ventilator tubing used, create incongruences that hinder comparisons and, by extension, the development of definitive recommendations. This is especially important considering the higher cost of some of the more efficient systems described in the literature.

See the Original Study on Page 1124

In this issue of Respiratory CARE, Berlinski and Willis ${ }^{2}$ test 4 different nebulizers ( 2 jet nebulizers, an ultrasonic, and a vibrating mesh nebulizer) in 4 different positions $(\mathrm{A}=$ proximal to the ventilator, $\mathrm{B}=$ proximal to the humidifier, $\mathrm{C}=$ at the $\mathrm{Y}$-piece, and $\mathrm{D}=30 \mathrm{~cm}$ before the Y-piece), with different loading doses and volumes of albuterol, to identify the ideal device and operating conditions for aerosol drug delivery in an in vitro pediatric model with bias flow. Although many of these variables have been independently studied for adults, their relationship has not been previously defined in a pediatric study.

They found that when placed at either position B or A (respectively, with albuterol $2.5 \mathrm{mg} / 3 \mathrm{~mL}$ ), the vibrating mesh nebulizer outperformed all the other nebulizers at all locations, a relationship also observed in a recent study by Ari et al. ${ }^{6}$ The efficiency of the vibrating mesh nebulizer was independent of fill volume. It delivered twice as much as the second highest, the ultrasonic nebulizer, and 5-6 times that of the jet nebulizers at that location.

Positioning any nebulizer at or near the Y-piece (positions $\mathrm{D}$ and $\mathrm{C}$ ) led to the least amount of drug being delivered to the endotracheal tube, regardless of nominal starting dose or fill volume. The jet nebulizers performed best when placed proximal to the ventilator or the humidifier. This finding is in agreement with prior data from the adult literature,,$^{7-9}$ yet differed from prior pediatric literature, ${ }^{6}$ possibly due to the use of varying amounts of external gas powering the nebulizers $(2.5 \mathrm{~L} / \mathrm{min}$ in prior study vs $6 \mathrm{~L} / \mathrm{min}$ in this study). Device specific analysis reveals that, although the Salter jet nebulizer did perform better at the humidifier and worst at the Y-piece (4.6\% vs 2.8 , respectively), the difference between the percent nebulized at the ventilator versus Y-piece is likely insignificant $(3.1 \%$ vs $2.8 \%)$.

Nebulizer type is known to be a major determinant of aerosol drug delivery during mechanical ventilation. Despite being less efficient than other nebulizer types, jet nebulizers are still commonly used to deliver aerosols in intubated patients. The nebulized dose in these devices may be as little as $50 \%$ of the starting dose, with resultant high residual volume, of up to $1.5 \mathrm{~mL} \cdot{ }^{10,11}$ Jet nebulizer efficiency varies with the pressure of the driving gas, different fill volumes, and presence or absence of bias flow. As illustrated in this study, moving the device back proximal to the ventilator or humidifier can increase its efficiency. Heating and humidified circuits have also been shown to reduce aerosol delivery by $>40 \%,{ }^{5}$ due to possible hygroscopic growth of the aerosol particles and presumed impaction or settling within the circuit. Intermittent operation during inspiration, although not studied in this series of experiments, has been shown to also greatly impact the delivery of aerosols in ventilator circuits. ${ }^{5}$ Continuous flow jet nebulization is being used less frequently, as most modern ventilators utilize breath-actuated jet nebulization.

Vibrating mesh nebulizers produce a relatively low velocity aerosol plume (compared to jet nebulizers), aerosolizing $90 \%$ of the nominal dose with minimal residual vol- 
ume $(0.1-0.3 \mathrm{~mL}),{ }^{12}$ but with generally larger particle sizes than jet nebulizers. As Berlinski and Willis ${ }^{2}$ illustrate in their Table 1, the vibrating mesh nebulizer is the lightest of all the nebulizers studied, has the least dead space, does not affect the temperature of the medication, does not add additional flow to the circuit, and does not require a separate external gas source. However, at first glance the apparent 10-fold difference in price does not seem to justify the above advantages. However, the cost over time to operate (120 treatments, or $30 \mathrm{~d}$ based on 4 treatments a day, as calculated) differs only by about 30 cents, making the vibrating mesh nebulizer overall cost-effective.

It is important to note, however, that this study does not report the range of particle sizes generated by the different devices, and hence make an inference regarding true "lung deposition," nor does it address efficacy in vivo. While it is human nature to champion for the most efficient system to be used at the bedside, it is not clear that the greater aerosol delivery seen in vitro with the vibrating mesh nebulizer leads to increased clinical efficacy at the beside. This is because, unlike converting an enteral dose of a drug to its bioavailable parenteral equivalent, aerosolized medications lack standard bioavailable dosing guidelines. In this respect, the clinical realm lags behind advances made at the bench, and continues its love affair with the jet nebulizer. The true usefulness of this study, however, lies not only in providing inter-device comparison for the pediatric population, but in the fact that it provides insight on how to best "optimize" aerosol delivery, given one's existing supplies. Using the data presented by Berlinski and Willis, ${ }^{2}$ one can choose to utilize different nebulizer po-

The author has disclosed no conflicts of interest.

Correspondence: Mohamed Mohsen Mansour MD, Division of Pulmonary and Critical Care Medicine, T17-040 Health Sciences Center, Stony Brook University Hospital, Stony Brook NY 11794-8172. E-mail: Mohamed.Mansour@stonybrookmedicine.edu.

DOI: $10.4187 /$ respcare. 02618 sitions, fill volumes, and/or loading dosages to enhance aerosol delivery to ventilated patients with one's current equipment. In doing so, the authors provide multiple options for overcoming the "jet lag."

Mohamed Mohsen Mansour MD

Division of Pulmonary and Critical Care Medicine

Stony Brook University Hospital

Stony Brook, New York

\section{REFERENCES}

1. Fuller HD, Dolovich MB, Posmituck G, Pack WW, Newhouse MT. Pressurized aerosol versus jet aerosol delivery to mechanically ventilated patients. Comparison of dose to the lungs. Am Rev Respir Dis 1990;141(2):440-444.

2. Berlinski A, Willis JR. Aerosol delivery by 4 different nebulizers placed in 4 different positions in a pediatric ventilator in-vitro model. Respir Care 2013;58(7):1124-1133.

3. MacIntyre NR, Silver RM, Miller CW, Schuler F, Coleman RE. Aerosol delivery in intubated, mechanically ventilated patients. Crit Care Med 1985;13(2):81-84.

4. Dhand R, Sohal H. Pulmonary drug delivery system for inhalation therapy in mechanically ventilated patients. Expert Rev Med Devices 2008;5(1):9-18.

5. Miller DD, Amin MM, Palmer LB, Shah AR, Smaldone GC. Aerosol delivery and modern mechanical ventilation: in vitro/in vivo evaluation. Am J Respir Crit Care Med 2003;168(10):1205-1209.

6. Ari A, Atalay OT, Harwood R, Sheard MM, Aljamhan EA, Fink JB. Influence of nebulizer type, position, and bias flow on aerosol drug delivery in simulated pediatric and adult lung models during mechanical ventilation. Respir Care 2010;55(7):845-851.

7. Ari A, Areabi H, Fink JB. Evaluation of aerosol generator devices at 3 locations in humidified and non-humidified circuits during adult mechanical ventilation. Respir Care 2010;55(7):837-844.

8. Hughes J, Saez J. Effects of nebulizer mode and position in a mechanical ventilator circuit on dose efficiency. Respir Care 1987; 32(12):1131-1135.

9. Quinn W. Effect of a new nebulizer position on aerosol delivery during mechanical ventilation. Respir Care 1992;37(5):423-431.

10. Fink JB. Humidity and aerosol therapy. In: Mosby's Respir Care Equipment. St Louis: Mosby-Elsevier; 2010:91-140.

11. Fink JB. Aerosol drug therapy. In: Egan's Fundamentals of Respir Care, 9th edition. St Louis: Mosby Elsevier; 2009:801-842.

12. Dhand R. Nebulizers that use a vibrating mesh or plate with multiple apertures to generate aerosol. Respir Care 2002;47(12):1406-1416. 